The majority of current chemotherapeutic regimens are based on intravenous administration. The most common and most frequent complication of chemotherapy is phlebitis, when given peripherally. The most feared complication of chemotherapy, such as in the case of doxorubicin, is extravasation. The extravasation itself is not always immediately obvious, so doctors and nurses should be aware of it in order to begin treatment as soon as possible. Late detection of extravasation can lead not only to immense damage of the affected area but also, in the worst case, even the inability to administer further cancer treatment. In the article we describe two clinical cases of extravasation and its therapy management. We try to show the economic differences between the use of dexrazoxane and surgery alone. It is obvious that the extravasation is not only a medical problem but it also has great economic impact. Nowadays there are new approaches to the treatment of doxorubicin extravasation, such as use of specific antidote dexrazoxane. The cost of a therapeutic dose of dexrazoxane is still the most negative aspect of the treatment.

Key words: doxorubicin, extravasation, prevention, chemotherapy.

Contemp Oncol (Pozn) 2014; 18 special issue DOI: $10.5114 /$ wo. 2014.40593

\section{Extravasation of doxorubicin - a dreaded complication of therapy}

\author{
Jindřich Kopecký ${ }^{1}$, Ladislav Slováček ${ }^{1}$, Peter Priester ${ }^{1}$, Vendelin Chovanec ${ }^{2}$, \\ Jiří Petera ${ }^{1}$, Otakar Kopecký ${ }^{3}$
}

${ }^{1}$ Department of Clinical Oncology and Radiotherapy, Charles University Hospital and Faculty of Medicine in Hradec Králové, Czech Republic

${ }^{2}$ Department of Radiology, Charles University Hospital and Faculty of Medicine in Hradec Králové, Czech Republic

${ }^{3}$ Clinical Oncology, Regional Hospital Náchod, Czech Republic

\section{Introduction}

The majority of current chemotherapeutic regimens are based on intravenous administration. Administration into the most peripheral veins is the most common means of cytotoxic agent application, so that in case of local toxicity the affected area would be minimal. An alternative to peripheral administration is application to the central vein (intravenous port). Proper application is associated with minimal side effects at the injection site. The most common and most frequent complication of chemotherapy is phlebitis. In the case of paravasal application or extravasation of cytotoxic agents into the surrounding tissue, tissue reaction ranges from just skin irritation to tissue necrosis, which at worst can even lead to loss of muscle function.

Cytotoxic agents can be divided into two main groups: cytostatics having a high risk of necrosis - also called vesicants, and cytostatics causing irritation of the vein without causing necrosis. Typical symptoms of vesicants are pain, burning, erythema, itching and swelling. The development of tissue necrosis following extravasation lasts several days and may continue for months because of drug diffusion into the adjacent tissue. Irritating substances usually cause only inflammatory reactions on site, itching, pain or phlebitis.

Management of extravasation/paravasation varies depending on the experience and treatment possibilities of each clinical department. The basic rule of treatment is the prevention of extravasation/paravasation (e.g. use of central venous access in cases of highly potent vesicants). Symptomatic treatment is based on the use of local or systemic anti-inflammatory drugs, which is not always sufficient in the case of vesicants. The extravasation/ paravasation itself is not always obvious immediately after administration of chemotherapy, and the patient often seeks medical attention (often through a general practitioner) as late as the first symptoms of extravasation appear. It is necessary that such patients are sent promptly to the clinical department where the cytotoxic agent was administered because it is responsible for the treatment of any side effects and has to be able to treat them or at least to prevent further worsening.

\section{Case report No. 1 - peripheral administration of anthracycline}

A 54-year-old patient treated for disseminated triple negative invasive ductal breast cancer was about to receive palliative chemotherapy in the composition of doxorubicin and cyclophosphamide. She was not treated with any internal comorbidities. In the past she underwent a cholecystectomy and she had a trauma accident with loss of distal and intermediate phalanges of three fingers on the right hand. Due to good performance sta- 
tus and biological age she was treated as an outpatient. Doxorubicin at a dose of $120 \mathrm{mg}$ was administered intravenously into the right cubital vein. During the administration of chemotherapy and after chemotherapy there was no sign of any extravasation, and the patient herself did not complain of any pain or other problems.

Ten days after the chemotherapy, the patient sought the general practitioner for swelling, redness and mild pain at the initial site of injection. The practitioner advised her to apply a cold pack to the affected area. The patient arrived at our department on the day of the next cycle of chemotherapy, i.e. 21 days after the first administration.

In the clinical finding, there was massive swelling of the entire right upper extremity and redness and superficial necrosis about $2 \times 1 \mathrm{~cm}$ without suppuration at the original injection site. Ultrasound of the right upper extremity did not show obvious signs of thrombosis or thrombophlebitis and there was no apparent fluid collection. The patient was given intravenous port, and a 12-day delay in the next series of chemotherapy was administered. During the administration of chemotherapy, as well as during the subsequent period, there were no alterations in white blood cell count or neutrophil counts.

Despite intensive local treatment (dressings, saline lavage, local application of Hyiodine ${ }^{\circledR}$ ) and systemic antibiotic therapy, there was progression in the extent of surface necrosis $(7 \times 7 \mathrm{~cm})$ and there was a newly identified
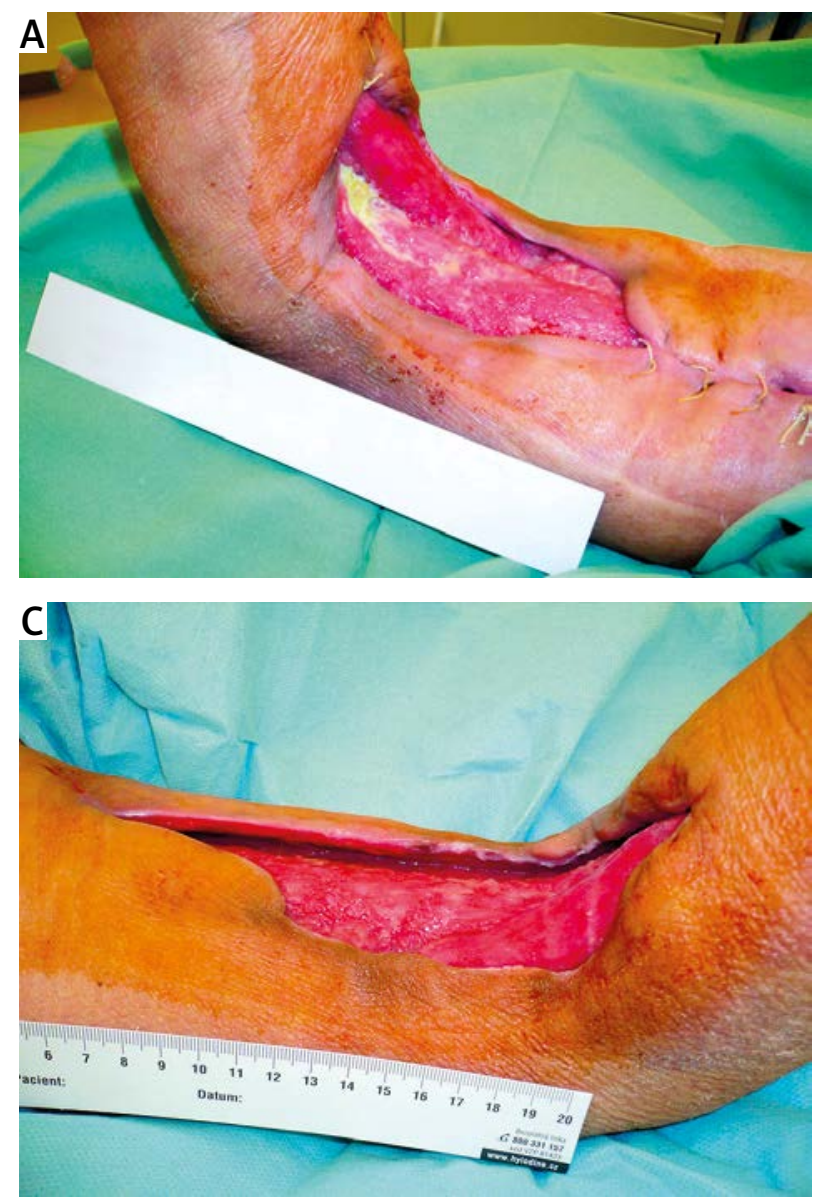

Fig. 1A-D. Peripheral extravasation subcutaneous necrotic pocket. Because of this progression and intermittent fever, the patient was hospitalized on $14^{\text {th }}$ February 2011. The antibiotic therapy was adjusted according to the cultivation from wounds, from which Staphylococcus aureus was repeatedly cultured. During hospitalization, the local therapy was carried on in cooperation with surgeons, but there was again progression in the local finding where the necrosis of musculus biceps brachii dominated, so necrectomy was indicated by surgeons (Fig. 1A). The operation (complete resection of necrotic musculus biceps brachii and extirpation of necrotic superficial fascia of the distal forearm) under general anaesthesia was performed on $13^{\text {th }}$ April 2011.

The patient was subsequently treated in the department of chronic wounds with topical application of Hyiodine ${ }^{\circledR}$ with slow wound granulation and reduction of wound size (Fig. 1B, C). The autotransplantation of skin under local anaesthesia, indicated by surgeons, was performed on $7^{\text {th }}$ July 2011. During the therapy and convalescence the cancer treatment plan was modified to monotherapy with capecitabine. The autotransplantation was successful and there has not been such massive loss of function of upper limb as we expected. The most visible problem seemed to be the fibrous scar (Fig. 1D). There was also apparent limitation of arm flexion, but with no affection of its function, which would have prevented the patient from performing everyday activities.
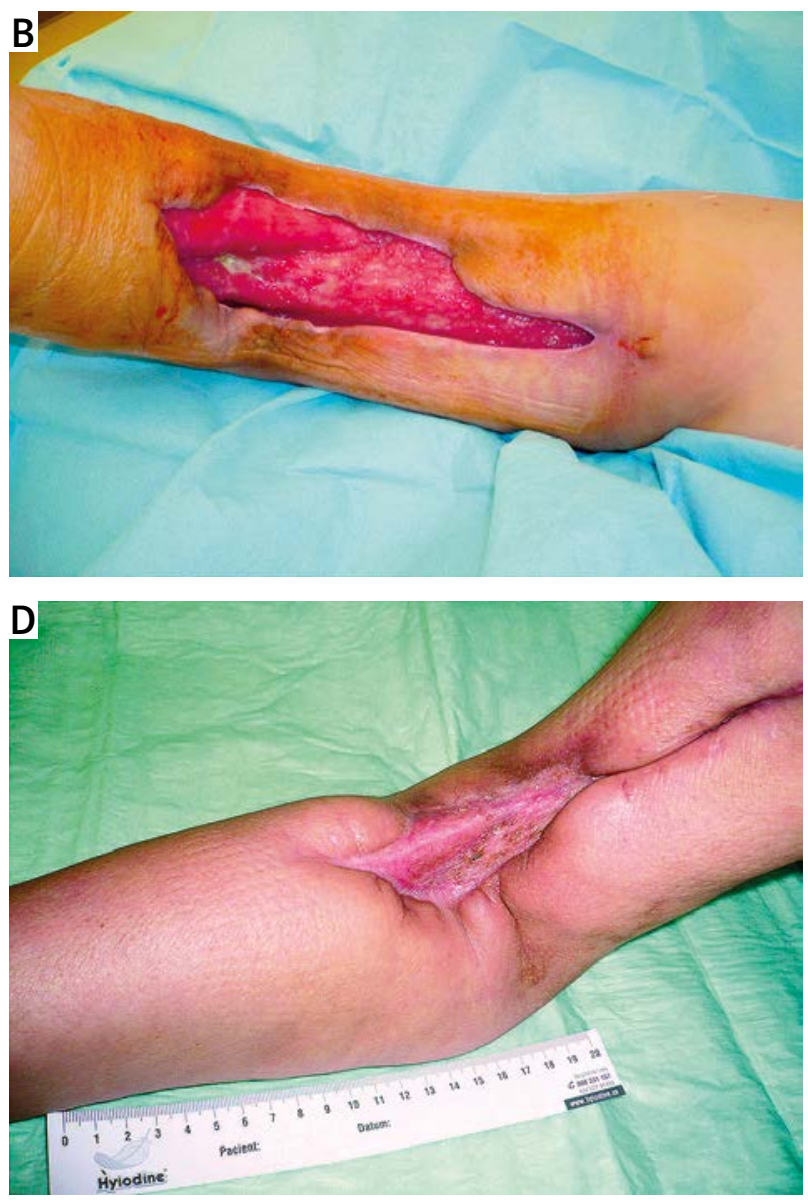


\section{Case report No. 2 - the central administration of doxorubicin}

In a 61-year-old patient diagnosed with a hormone dependent, low-differentiated, left-sided ductal breast cancer (pT1cpN1M0) treated with mastectomy and axillar lymph node dissection, adjuvant chemotherapy (doxorubicin with cyclophosphamide and then taxol twelve times a week) was established.

Because of peripheral venous deficiency, the implantation of an intravenous port was indicated. The implantation was performed on $22^{\text {nd }}$ March 2010, with RTG control of the port on the following day. The first chemotherapy was given two days later. At the beginning of administration, clinical signs of extravasation were observed in the surrounding port area (Fig. 2A). The application was immediately stopped and about $8 \mathrm{ml}$ of extravasation was aspirated.

The patient was hospitalized and local treatment (cooling) and systemic administration of antibiotics was started, and the function of the intravenous port was examined by a radiologist. He found disruption of the membrane integrity. Because of the high risk of chemical necrosis, excision of the intravenous port was performed on $26^{\text {th }}$ June 2010. A subcutaneous pocket was described locally around the venous port, filled with white matter (Fig. 2B). The dominating sign was locally elevated temperature. Two drains were temporarily left in the wound area and further
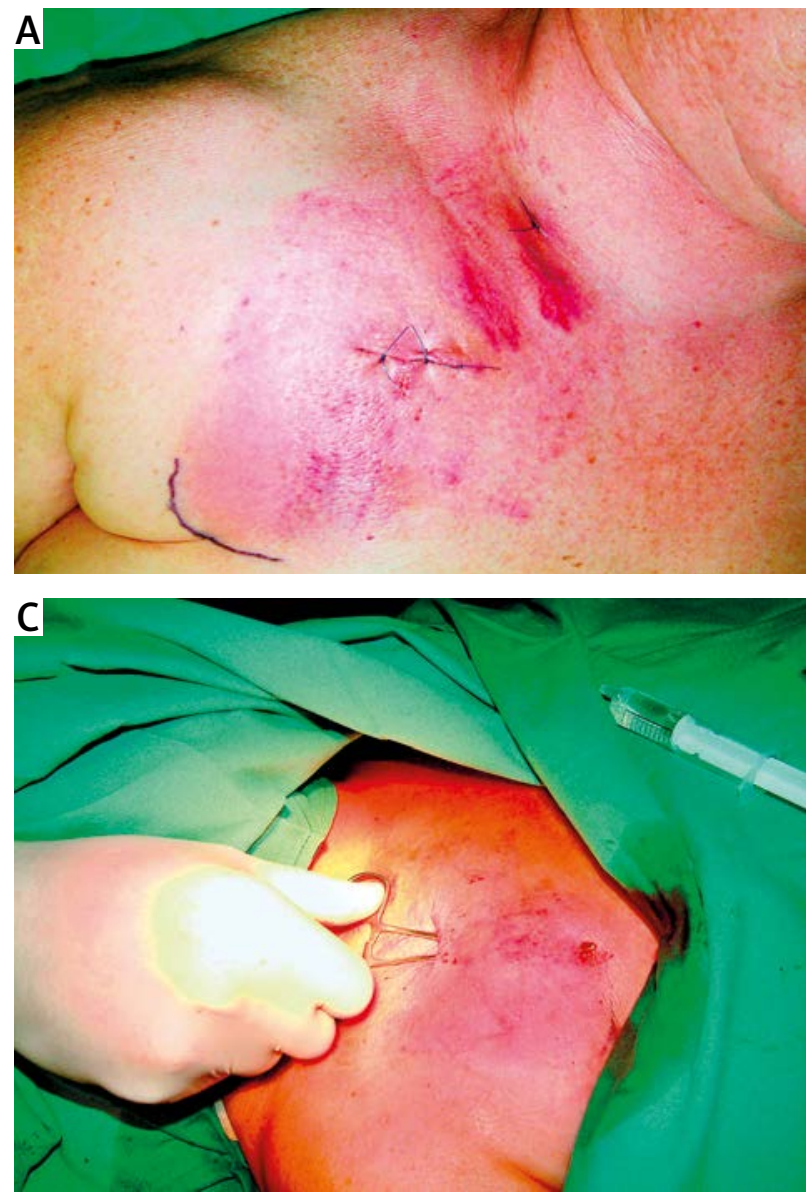

Fig. 2A-D. Central extravasation local therapy (cooling and daily dressing) and systemic antibiotic treatment was continued (Fig. 2C). The patient was examined by surgeons and no necessity for surgical intervention was found. In order to prevent delay of chemotherapy, further treatment was given into a peripheral vein. The patient was discharged to continue treatment as an outpatient.

Ten days after the excision, a physical examination was dominated by local hyperpigmentation and palpation sensitivity of the affected site (Fig. 2D). The oncology treatment was not delayed and the patient completed all remaining cycles without complication. The patient remains on hormonal treatment. There is persisting scarred contracture and hyperpigmentation at the extravasation site, but there is no loss of muscle function or other limitations.

\section{Discussion}

Extravasation/paravasation is defined as the leak of fluid from blood vessels. The frequency of this complication is estimated between $0.1 \%$ and $6 \%[1,2]$. The consequences of extravasation are dependent on the type, concentration and quantity of extravaseted chemotherapeutic agents. According to tissue reactions, the cytotoxic agents can be divided into substances causing blisters (vesicant) and irritant substances.

Anthracyclines belong to the most potent group of vesicants. In the case of significant extravasation, as in our
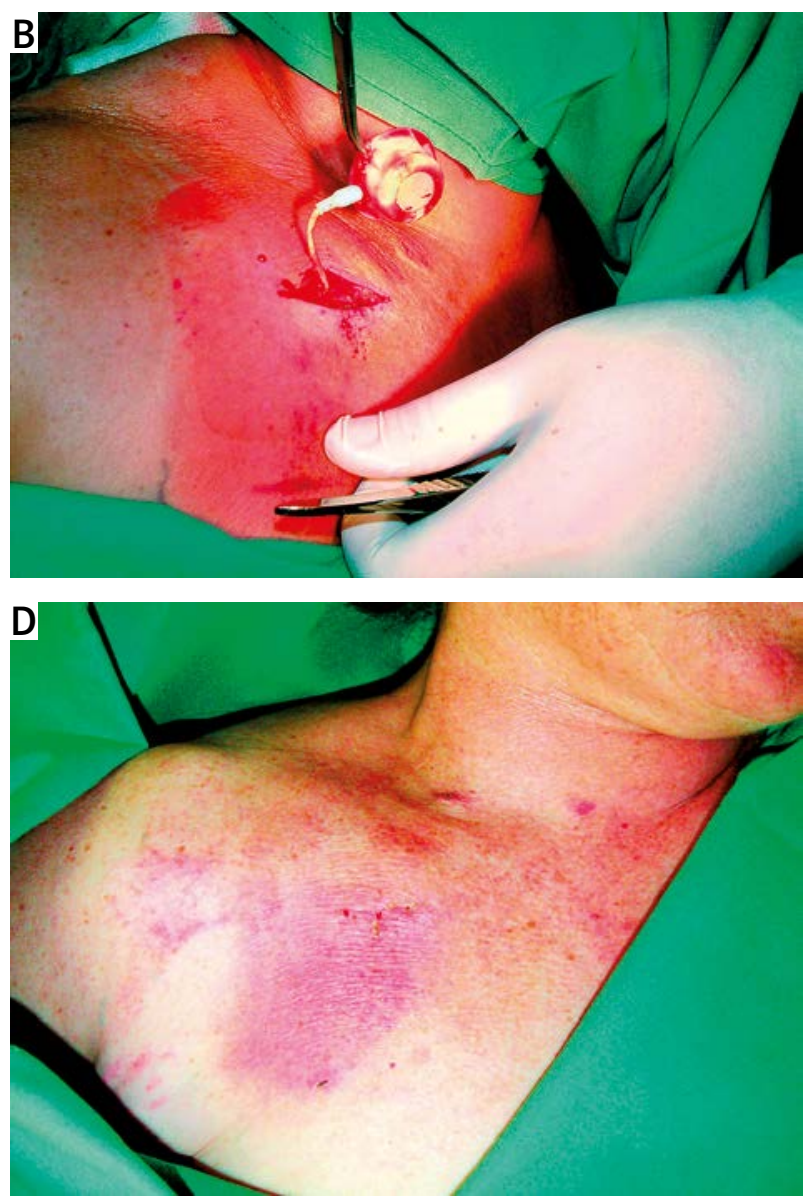
cases, necrosis or ulceration of the affected area can occur. The pathogenesis of necrosis caused by anthracycline is caused by their ability to bind to DNA intracellularly in the extravasation site, which may lead to cell death. The subsequent release of DNA complexes with anthracyclines from damaged cells leads to further uptake by the surrounding healthy cells, so it can evoke further tissue damage $[3,4]$.

Prevention of extravasation is the main and most important part of the care of patients during chemotherapy administration. Primarily there are the general recommendations and guidelines of oncology societies [5, 6], and then each oncology department has its own guidelines tailored to their abilities and equipment regarding how to proceed if extravasation occurs.

Furthermore, it is essential that medical and nursing staff, who are responsible for administering chemotherapy, are regularly trained and educated in this field. Generally every doctor and nurse should be familiar with the main principles of prevention:

1. Do not apply cytotoxic drug into the veins if there was application of chemotherapy above this site in the previous 48 hours.

2. Do not apply cytotoxic drugs into apparently fragile veins or into extremities with obvious lymphedema or neurological weakness.

3. Do not use veins of the dorsal hand for chemotherapy application (there is a danger of greater tissue damage in the case of extravasation).

4. Check the position of the venous catheter both prior to and after chemotherapy application by flushing with saline solution. An important part of prevention is the education of patients about potential risks and the need for prompt intervention in the case of extravasation.

The prevention of complications by using an implantable central venous port is dependent on the right and proper techniques of the implantation itself. There are four main causes of extravasation by intravenous port:

- poorly stabbed port needle or dislocation of the port needle,

- thrombosis or fibrin clot formation in the catheter,

- perforation of superior vena cava,

- broken catheter [7].

Patients with this kind of venous access should be checked for right function at regular intervals after initial application of a cytotoxic agent, even if the venous port is not used for a long time.

There are two main approaches in the surgical treatment of extravasation:

1. Early surgical intervention with extensive debridement within the first 24 hours or up to 1 week with delayed suture, followed by plastic reconstruction [8].

2. Local conservative treatment and, in the case of progression, surgical intervention [9].

Historically, many treatment options have been examined in an attempt to mitigate the devastating effects of anthracycline extravasation and reduce or even replace surgical treatment. These options include cooling the injured area, application of saline lavage, hyperbaric oxygen therapy [10], local application of growth factors [11], local application of dimethyl sulfoxide [12] and systemic application of dexrazoxane $[13,14]$.

The only treatment option proven to reduce the necessity of surgical intervention after anthracycline extravasation has been dexrazoxane (Savene ${ }^{\circledast}$, Totect ${ }^{\circledR}$ ). Its efficiency has been verified by an open single-arm multicentre study and a multicentre retrospective survey in 29 Belgian hospitals $[15,16]$.

Dexrazoxane is an iron chelator, which absorbs the free radicals induced by anthracyclines. Furthermore, it is assumed that the protective effect of dexrazoxane is based also on the inhibition of topoisomerase II, abrogating the mechanism of action of the anthracyclines [17].

The recommended dosage of dexrazoxane is $1000 \mathrm{mg} /$ $\mathrm{m}^{2}$ on the first and second days and $500 \mathrm{mg} / \mathrm{m}^{2}$ on the third day. The prerequisite for the effectiveness of this treatment option is the administration of the first dose up to 6 hours after extravasation into a large-calibre vein at the contralateral side [18].

The cost of a therapeutic dose of dexrazoxane is the main negative aspect of the treatment itself. In the pharmacological market, cheaper dexrazoxane (Cardioxane ${ }^{\circledR}$ ) is available but with no indication for use in the case of extravasation. Cardioxane ${ }^{\circledR}$ differs from Totect ${ }^{\circledR}$ or Savene ${ }^{\circledR}$ only in the type of solvent (Ringer's solution in contrast with sterile water). The cost of 500 mg of Cardioxane ${ }^{\circledR}$ in the Czech Republic is eight times cheaper than Totect ${ }^{\circledR}$. Spanish authors Arroyo et al. calculated the savings made by using Cardioxane ${ }^{\circledR}$ instead of Savene ${ }^{\circledR}$ to be $€ 8685$ for the total therapeutic dose [19].

The total cost of treatment of extravasation without the use of dexrazoxane has not been established yet. The total cost of the treatment can vary depending on the severity of extravasation. In our patient with peripheral extravasation it was $€ 8062$, and it was $€ 1262$ in the second case. This amount consists of direct costs such as antibiotic therapy, medical supplies (gauze sponges, dressings, tapes etc.), hospitalization, surgery (necrectomy, plastic surgery, respective excision of intravenous port), application of Hyiodine ${ }^{\circledR}$, and laboratory analysis, but indirect costs were not included, such as loss of work, social disadvantage and the therapeutic effects of modified therapy.

The incidence of cancer in the world is rising and so is the number of people receiving some kind of cytostatic therapy. Therefore, any doctor coming into contact with such patients must have basic knowledge of the adverse effects of cancer therapy, especially effects that can be prevented or successfully managed with early detection. This is also true for complications like extravasation or paravasation. Late detected extravasation of doxorubicin can lead not only to immense damage of the affected area but also, in the worst case, the inability for further cancer treatment to be administered.

The best method to prevent complications such as extravasation is its own prevention. But even with the best prevention, complications can occur. In the case of anthracycline extravasation, early detection is imperative including immediate confinement of the patient in the hospital where the chemotherapy had been administered. Every 
oncology department then follows its guidelines for extravasation/paravasation. The possibility of application of dexrazoxane should be considered as part of such guidelines because it is the only proven antidote to anthracyclines, which prevents surgical intervention and treatment delay significantly.

\section{Authors declare no conflict of interest.}

\section{References}

1. Bertelli G. Prevention and management of extravasation of cytotoxic drugs. Drug Safety 1995; 12: 245-55.

2. Dorr RT. Antidotes to vesicant chemotherapy extravasations. Blood Rev 1990; 4: 41-60.

3. Luedke DW, Kennedy PS, Rietschel RL. Histopathogenesis of skin and subcutaneous injury induced by Adriamycin. Plast Reconstr Surg 1979; 63: 463-5.

4. Mross K, van der Vijgh WJ, Gall H, Boven E, Pinedo HM. Pharmacokinetics and metabolism of epidoxorubicin and doxorubicin in humans. J Clin Oncol 1988; 6: 517-26.

5. Pan-Birmingham NHS: Guidelines for the Management of Extravasation. Available at: http://www.birminghamcancer.nhs.uk/ uploads/document_file/document/4e1bf958358e982bc500080f/ management_of_extravasation_version_2.0.pdf.

6. Jacobson JO, Polovich M, McNiff KK, Lefebvre KB, Cummings C, Galioto M, Bonelli KR, McCorkle MR; American Society of Clinical Oncology; Oncology Nursing Society. American Society Of Clinical Oncology/Oncology Nursing Society chemotherapy administration safety standards. J Clin Oncol 2009; 27: 5469-75.

7. Schulmeister DL, Camp-Sorrell D. Chemotherapy extravasation from implanted ports. Oncol Nurs Forum 2000; 27: 531-8.

8. Heitmann C, Durmus C, Ingianni G. Surgical management after doxorubicin and epirubicin extravasation. J Hand Surg 1998; 23: 666-8.

9. Tsavaris NB, Komitsopoulou P, Karagiaouris P, Loukatou P, Tzannou I, Mylonakis N, Kosmidis P. Prevention of tissue necrosis due the accidental extravasation of cytostatic drugs would and conservative approach. Cancer Chemother Pharmacol 1992; 30: 330-3.

10. Monstrey SJ, Mullick P, Naranyanan K, Ramasastry SS. Hyperbaric oxygen therapy and free radical production: an experimental study in doxorubicin (Adriamycin) extravasation injuries. Ann Plast Surg 1997; 38: 163-8.

11. Ulutin HC, Güden M, Dede M, Pak Y. Comparison of granulocyte-colony stimulating factor and granulocyte macrophage-colony stimulating factor in the treatment of chemotherapy extravasation ulcers. Eur J Gynaecol Oncol 2000; 21: 613-5.

12. Lawrence HJ, Walsh D, Zapotowski KA, Denham A, Goodnight SH, Gandara DR. Topical dimethyl sulfoxide may prevent tissue damage from anthracycline extravasation. Cancer Chemother Pharmacol 1989; 23: 316-8.

13. Schrijvers DL. Extravasation: a dreaded complication of chemotherapy. Ann Oncol 2003; 14 (Suppl 3): 26-30.

14. Wickham R, Engelking C, Sauerland C, Corbi D. Vesicant extravasation part II: Evidence -based management and continuing controversies. Oncol Nurs Forum 2006; 33: 1143-50.

15. Mouridsen HT, Langer SW, Buter J, Eidtmann H, Rosti G, de Wit M, Knoblauch P, Rasmussen A, Dahlstrom K, Jensen PB, Giaccone G. Treatment of anthracycline extravasation with Savene (dexrazoxane): results from two prospective clinical multicentre studies. Ann Oncol 2007; 18: 546-50.

16. Fontaine C, Noens L, Pierre P, De Grève J. Savene ${ }^{\circledR}$ (dexrazoxane) use in clinical practice. Support Care Cancer 2012; 20: 1109-12.

17. Schulmeister DL. Totect: a new agent for treating anthracycline extravasation. Clin J Oncol Nurs 2007; 11: 387-95.

18. Langer SW, Sehested M, Jensen PB, Buter J, Giaccone G. Dexrazoxane in anthracycline extravasation. J Clin Oncol 2000; 18: 3064.
19. Arroyo PA, Perez RU, Feijoo MA, Hernandez MA. Good clinical and cost outcomes using dexrazoxane to treat accidental extravasation of epirubicin. J Cancer Res Ther 2010; 6: 573-4.

\section{Address for correspondence}

\section{Jindřich Kopecký}

Department of Clinical Oncology and Radiotherapy

Charles University Hospital and Faculty of Medicine in Hradec Králové Sokolska 581

50005 Hradec Králové, Czech Republic

e-mail: kopecjin@fnhk.cz

Submitted: 4.05 .2012

Accepted: $\quad 7.08 .2012$ 